\title{
Unexpected Situations in Service Robot Environment: Classification and Reasoning Using Naive Physics
}

\author{
Anastassia Küstenmacher ${ }^{1}$, Naveed Akhtar ${ }^{1}$, Paul G. Plöger ${ }^{1}$, \\ and Gerhard Lakemeyer ${ }^{2}$ \\ 1 Department of Computer Science, Bonn-Rhein-Sieg University of Apply Science, \\ Sankt Augustin, Germany \\ \{anastassia.kuestenmacher, naveed.akhtar, paul.ploeger\}@h-brs.de, \\ 2 Knowledge-Based Systems Group, RWTH Aachen University, Aachen, Germany \\ gerhardakbsg.rwth-aachen.de
}

\begin{abstract}
Despite perfect functioning of its internal components, a robot can be unsuccessful in performing its tasks because of unforeseen situations. Mostly these situations arise from the interaction of a robot with its ever-changing environment. In this paper we refer to these unsuccessful operations as external unknown faults. We reason along the most frequent failures in typical scenarios which we observed during real-world demonstrations and competitions using our Care-O-bot III robot. These events take place in an apartment-like environment.

We create four different - for now adhoc - fault classes, which refer to faults caused by a) disturbances, b) imperfect perception, c) inadequate planning or d) chaining of action sequences. These four fault classes can then be mapped to a handful of partly known, partly extended fault handling techniques.

In addition to existing techniques we propose an approach that uses naive physics concepts to find information about these kinds of situations. Here the naive physics knowledge is represented by the physical properties of objects which are formalized in a logical framework. The proposed approach applies a qualitative version of physical laws to these properties to reason about the fault. By interpreting the results the robot finds the information about the situations which can cause the fault. We apply this approach to scenarios in which a robot performs manipulation tasks (pick and place). The results show that naive physics hold great promises for reasoning about unknown external faults in the field of robotics.
\end{abstract}

Keywords: faults in robotics, unexpected situations, naive physics.

\section{Introduction}

Robots, operating in the open world, outside of lab conditions, may face situations where they are not able to perform their task successfully. These situations occur instantaneously, are sporadic in nature and are caused by interaction with the environment.

In the RoboCup@Home League the main focus is to enable a service robot to perform household chores. Clear or set the table, clean up the apartment, fetch and deliver objects: these are typical tasks for a service robot. To perform them in a desired manner in an uncertain environment, the robot needs the ability to handle unforeseen situations.

S. Behnke et al. (Eds.): RoboCup 2013, LNAI 8371, pp. 219-230, 2014.

(C) Springer-Verlag Berlin Heidelberg 2014 


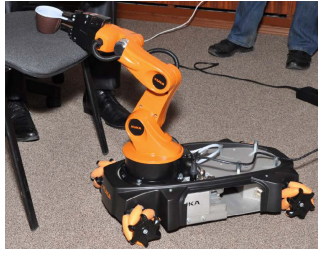

(a) Scenario 1

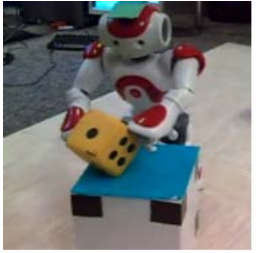

(b) Scenario 2

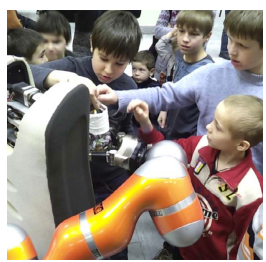

(c) Scenario 3

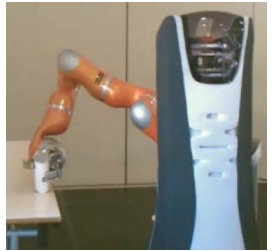

(d) Scenario 4

Fig. 1. Examples of unexpected situations

To illustrate the range of these situations we will consider the following scenarios:

\section{Scenario 1: Collision with unknown obstacle}

A service robot needs to build a map of a domestic environment using its sensors (e.g. cameras and laser rangefinders). How should it react when colliding with an unexpected obstacle like a glass door or a desk of a table which may not be detected by any of its perception devices (Fig. 1. (a))?

\section{Scenario 2: Impact of inaccurate action performance}

A robot has to transfer a die from the floor to a table (Fig. 1. (b)). While bending down, the robot kicks the die accidentally and as a consequence grabs the die wrongly (i.e. at two opposite corners instead of at two parallel faces). The wrong grip causes the die to fall from the table while being placed. How can the real cause of the fault, namely kicking the die, be identified?

\section{Scenario 3: Pushed/held by an external actor}

A robot has to travel from one place to another. During its movement it is pushed or held by an external actor (Fig.1 (c)). Hence the robot can not reach its desired location. How can we enable the robot to identify the external fault so that it can reach the desired location?

\section{Scenario 4: Imprecise knowledge about properties of an object}

In this scenario a robot has to place a bottle on the table. It should stand stable in the final state, but it does not and falls. It is possible that the robot was not holding the bottle properly before releasing it on the table. The robot's gripper with the bottle inside could be slightly inclined. How may the robot learn from this example and avoid this fault in the future (Fig.1 (d))?

Note that in the described situations all of the robot's internal components are working perfectly fine. Here the causes of failing the task originate from outside and cannot be explained by the robot's knowledge. Therefore, these situations are termed as exogenous causes of failure or external unknown faults. In our scenarios, external unknown faults refer to the existence of an external impact, partially known properties of objects and unmodeled consequence of executed actions.

The majority of robots utilize the traditional fault handling approaches which work well under the assumptions of known a priori faults. However, their performance substantially drops if they are subject to unexpected events. Unfortunately, we cannot expect that all possible fault sources have already been encountered in practice.

Based on our research and on the studies conducted by other researchers we developed a method to handle some of these situations, classified them and assigned 
appropriate techniques to each class individually. For now we select four fault classes and enumerate a set of techniques for solving them.

Our reasoning approach uses naive physics knowledge and a qualitative version of physical laws to find reasons of the occurrence of the detected unexpected situations, namely external unknown faults. These situations occur when the behavior of the objects in the robot environment deviates from its expected values.

\section{Classification of Unexpected Situations}

Traditional model-based fault diagnosis techniques focus on improving the reliability of robots in certain well-known fault situations. The widely known autonomous robot Shakey [1] was already equipped with model-based fault detection capabilities. Shakey's execution monitoring system PLANEX monitored a sequence of actions, provided by the STRIPS planner for accomplishing a required task. After executing each action the monitor checked for inconsistencies between observations and the robots internal representation. These inconsistencies indicated a faulty plan but could not identify the cause of fault.

Despite successfully implementing the model-based algorithms, they do not diagnose exogenous causes of failure. Nevertheless, model-based techniques allow a robot to detect unexpected situations if its nominal behavior is modeled. Pettersson [2] presents a model-free approach to execution monitoring. He assumes that instead of having a model with predefined faulty states, only the nominal behavior of a robot is given. Therefore the detection of faults is performed based on the comparison between observed and expected behaviour.

Steinbauer [3] notes that most of the faults that can be observed during a RoboCup may be avoided by a more careful design of hardware devices and software components. He based his observations on fault-reports from the RoboCup competitions, especially when systems operate in conditions similar to lab environments and chances for unexpected situations are reduced to a minimum. But he also states that unexpected situations could not be dealt so easily.

The researches dealing with unexpected situations refer to them with keywords like errant expectations [5], unexpected events [6], exogenous events [4] and external unknown faults. In the scenarios mentioned in section 1 and in many other similar situations, a successful completion of robot's tasks suffers from many exogenous factors. Such kind of faults cannot be solved by any of the existing fault handling techniques.

At the same time each of the described scenarios may on its own be handled using particular algorithms. For instance, Ueda [7] proposes to re-plan the robotic task in the case of the robot being pushed by an external actor as in scenario 3. Mendoza [8] utilizes a hidden Markov model-based model for detecting motion interference events in mobile robots. He successfully tests his approach for three types of motion interference: collision with undetected obstacles (scenario 1), held by a human (scenario 3 ) and stuck wheels. In his research Sundvall [6] uses the other estimation-based method extended Kalman filter (especially successful for the navigation problems as in scenario 1) for detecting unexpected events, such as a collision with an unexpected obstacle. Gspandl [4] uses a belief-based management system to reason about the sequence of 
past executed actions of the robot and their results. This algorithm can be suitable for solving the problem of scenario 2 .

Although none of these algorithms can be a common solution for the problem of handling all different unexpected situations together, individually these situations may be solved. Therefore, it makes sense to evaluate these algorithms and enumerate assumptions for them as well. Based on these assumptions we suggest to classify various exogenous events into different classes, and then associate each class with one or more appropriate fault handling algorithms. Based on existing methods and observations from the given scenarios we propose to group the unexpected situations into four major classes:

1. Inadequate description of planner operator (e.g. unsuccessful completion of action because of its insufficient description)

2. External disturbance (e.g. not reaching expected location because of being pushed/ held by external actor)

3. Imprecision of perception devices (e.g. delusion of robot due to glass door)

4. Consequence of previous inaccuracy (e.g. incorrect release of die because of inaccurate grasping)

Our contributions in this field have been made in reasoning about faults from the inadequate description of planner operator class. We mainly focussed on actions which deal with the release of objects (like scenario 2) and do not consider the presence of external agents in the environment of robots. We assume natural physical phenomena as the only cause of external faults and propose to utilize naive physics for reasoning about these situations.

\section{Naive Physics}

In [9], Hayes proposes to formalize everyday knowledge of the physical world into a declarative symbolic theory. In principle, such a theory represents the naive physics knowledge which can be used for commonsense reasoning about everyday physical phenomena. This proposal of Hayes is one of the widely acclaimed ideas in AI, but it has never been truly followed [10]. For commonsense reasoning, the literature in AI is mostly concerned with what is more appropriately called qualitative physics [11]. In contrast to naive physics, qualitative physics concentrates on reasoning programs rather than knowledge representation. Furthermore, qualitative physics emphasizes on restricted systems rather than daily life physical phenomena.

One approach of representing commonsense physical knowledge, is the methodology of microworlds [10]. In his work [10] Davis proposes to structure the knowledge in microworlds, where "a microworld is an abstraction of a small part of physical interactions, sufficient to support some interesting collection of inferences". Component-based electronics [11], rigid object kinematics and dynamics are few examples of microworlds. Such structuring of the knowledge makes its formalization easier. In comparison to Hayes' proposal, any knowledge formalized using the microworlds approach is more inferencing oriented. It also implies that keeping in view the collection of inferences to be supported by a microworld, we can limit the scope of the knowledge to 
be formalized for that microworld. This aspect of the microworlds approach makes it more practical in comparison to the original proposal of Hayes.

Reasoning about the unknown external faults in robotics requires the ability to reason about physical phenomena encountered in our daily life. As noted in [12], approaches in qualitative physics (QSIM [13] and qualitative process theory [14] etc.) are inadequate for such reasoning. On the other hand, it is also true that in current AI literature there does not exist any large scale formalization of daily life physical phenomena (formalized in a naive physics theory or structured into microworlds) which can be used directly for unknown external faults. Therefore, we use insights from the works referenced in this section to formalize a small body of naive physics knowledge for reasoning about unknown external faults in robotics. Our proposed approach illustrates how naive physics knowledge can be formalized in a way that we can benefit from it for reasoning about unknown external faults.

\section{Naive Physics Approach for Unknown External Faults}

When a fault is detected by a robot because of unforeseen situations in its environment, it needs to find out the information about the cause of the fault to avoid it in the future.In the proposed approach, this information is produced in the form of hypotheses, which describe the possible situations that could have caused the detected fault. Each of these hypotheses is generated as a state of the object, whose parameters are the properties of that object. These properties are representative of naive concepts behind them which are defined over extended time. Thus, the states produced by the reasoning process characterize the behavior of the object over an extended time. This allows the robot to achieve the goal of finding the cause behind a detected fault with minimal information and computation.

In the reasoning scheme (Fig. 2]), the hypotheses only represent those situations which could result in the observed behavior of the object under the influence of physical phenomena e.g. gravity and friction. In a detected fault, the physical phenomena cause the object to achieve a final state that is not the goal state for the robot. The reasoning scheme uses this final state of the object and the physical phenomena to trace the possible situations that could have caused the detected fault.

\subsection{Proposed Scheme}

The name of each module in Fig. 2 represents its function in the reasoning scheme. We describe each of the modules with a running example of scenario 2 in section 1 . The proposed scheme focuses on finding the reasons for unknown faults which are associated with the current action of the robot (i.e. releasing the die). 1

Fault Categorizer. In our example the robot detects an unknown fault when it finds that its action was unsuccessful. This fact becomes known to the robot when it detects that a relation on (table, die) is unsatisfied in the effects of the action performed

\footnotetext{
${ }^{1}$ The description of modules uses Prolog syntax to show the relations and logical sentences used for the scenario.
} 


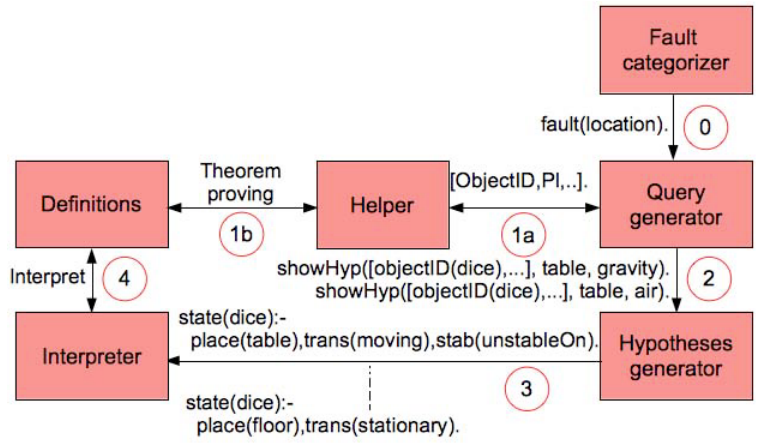

Fig. 2. Scheme of reasoning

by the robot. Based on this unsatisfied relation, the fault categorizer categorizes the detected fault. The categories of the detectable faults are defined a priori (see section 4.2). In the running example, the category of the fault is chosen to be location. In this work we assume that the detection of the fault has already been made by the robot and the fault categorizer has the ability to categorize any of the unsatisfied relations in the effects of robot's actions. The transmission of the signal from the fault categorizer is shown as step 0 in the scheme of Fig. 2.

Query Generator. The signal of the fault occurrence is passed on the query generator along with the category of the fault. In Fig. 2, fault (location) shows this signal. The query generator uses this signal to automatically generate relevant queries for the hypotheses generator.

Helper. The process of the generating of queries requires the knowledge of the final state of the object. That is, the state of the die being on the floor. This knowledge is made available to the query generator by a helper. The helper helps in defining the final state of the object by filling in the values of a list of properties, which is transmitted to it by the query generator (step 1a in the Fig. 2.). The helper also provides the query generator with a certain value of a property of the object that is involved in the detection of the fault. In our example, the constant symbol table represents this value. This value is decided from the failed relation on (table, die), which indicates that the desired place 'table' was not achieved by the object as an effect of the robot's action.

Definitions. The helper uses the observations of the robot and the definitions of properties stored in the module named as definitions, to define the final state of the object. The definitions of the properties are formalized in the form of a logical framework (see section 4.3). In the work presented here, the job of the helper is performed manually by using logical theorem proving to define the final state of the object. Therefore, step $1 \mathrm{~b}$ in Fig. 2 is indicated as theorem proving.

Hypotheses Generator. In step 2, the query generator transmits the queries to another module which is called hypotheses generator. This module generates hypotheses about the situations that could have caused the detected fault. These hypotheses are represented as states of the concerned object. Each of these states is a conjunction of possible values of relevant properties of the object. These values are constraint by the qualitative version of physical laws used by the hypotheses generator. The physical laws let the module generate only those states of the object which can result in its 
observed final state under the phenomenon represented by the physical law. We define the physical laws by simple Prolog clauses which can generate lists of values of properties for the hypotheses. For our example, the clause shown in Fig. 3 (a) serves the purpose of the gravitational law for the hypotheses generator. Here, when a relevant query is posed to the hypotheses generator it automatically uses this clause to generate hypotheses. Another clause (not shown here) used by the hypotheses generator in this scenario is regarding the phenomenon of motion due to air. Both the clauses are used by the module to generate only relevant hypotheses for the occurred fault.

Interpreter. The generated hypotheses are received by an interpreter (step 3 in the Fig. 2]). In addition to receiving the hypotheses, this module performs the function of interpreting the hypotheses using the definitions of the properties in the definitions module. This interpretation (i.e. step 4) results in generating the information that can be used by the robot to avoid the occurrence of the detected fault in the future. In the work presented here, the function of this module is performed manually and the interpretation only represents the meanings of the hypotheses in natural language. This is done in order to show that meaningful information about the occurred situation can be extracted from naive physics knowledge using the proposed approach.

\subsection{Ontology}

In the scheme shown in Fig. 2, the query generator and the hypotheses generator require the knowledge of relevant properties of the object and relevant physical laws to perform their functions effectively. This knowledge is embedded in these modules in the form of an ontology, shown in Fig. 3. (b). This ontology is mainly derived from a list of properties of physical objects (made of substances) proposed in substance schema [15]. These properties represent the concepts used by naive reasoners to reason about daily life physical phenomena.

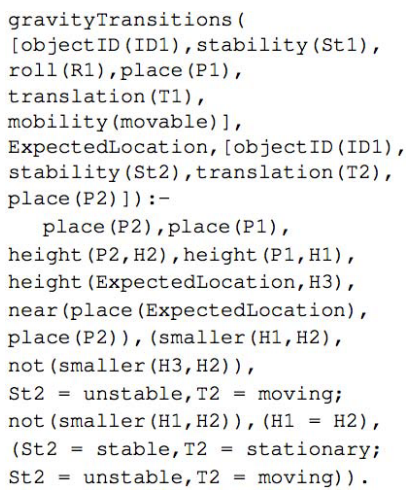

(a) Hypotheses

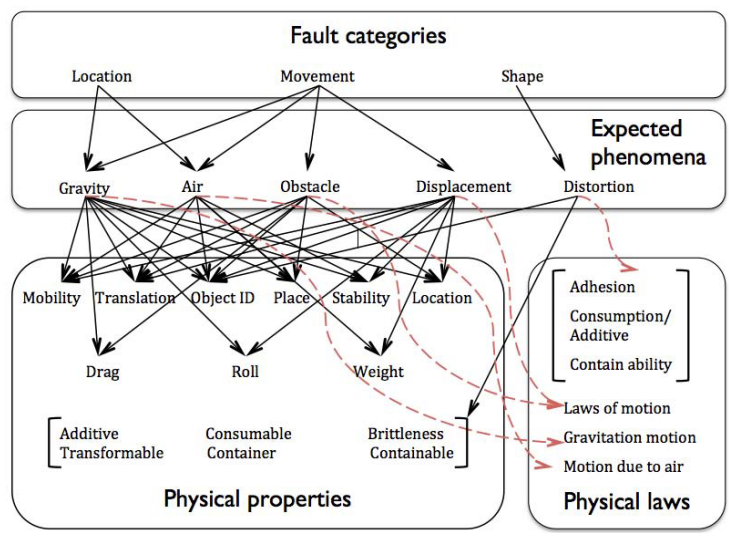

(b) Ontology

Fig. 3. Example of hypotheses and ontology used in reasoning process

The fault categories shown in the ontology are formed by considering the tasks which can be performed by the robots for which the ontology is being created. In this work, 
we restrict these broad categories only to those shown in the Fig. 3. (b), because we only consider the robots which can perform simple manipulation tasks (e.g. picking and dropping/placing objects). In such tasks, faults can be detected when the robot is unable to achieve the desired location of the object. Or, when the object behaves unexpectedly because of some movement, or when its shape gets distorted. We do not claim here that these categories are enough for all unknown faults for the considered robots. However, it is easy to see that these categories cover a large range of situations that can result in detectable faults for such robots.

The ontology associates different categories of faults to different expected phenomena which can cause these types of faults. These phenomena provide links between the physical laws and the physical properties of objects which are relevant for the expected phenomena. In our scheme the list of relevant properties is used by both the query generator and the hypotheses generator. However, the physical laws are only utilized by the hypotheses generator to generate possible hypotheses.

\subsection{Framework}

We formalize the definitions of properties of objects in the form of a logical framework. This framework is meant to illustrate how the properties of objects can be defined such that the definitions are useful for reasoning about unknown external faults. The mention framework only deals with the fault category location. We propose formalization of different frameworks for different fault categories. Such structuring of naive physics knowledge enables easy identification of physical interactions which are sufficient to support inferencing for their relevant fault categories. A detailed account on the developed framework can be found in [16], here we only summarize the framework by illustrating its important aspects.

The developed framework is meant only for solid objects. It considers the geometry to be $\mathbf{R}^{3}$, a subset of which is occupied by any object in the domain. Also, the shape of any object is equal to the closure of its interior and the object itself is considered as a primitive entity. All these notions are the same as those used in the framework proposed in [12]. However, our work differs greatly from Davis's work in dealing with time and physics. Our framework captures the notion of time by the concepts of intervals and instants, where an interval is just a finite set of instants. The framework formalizes naive physics concepts in the form of definitions of predicates and functions in first-order logic. Those concepts which depend on time, have explicit mentioning of respective temporal notions in the definitions. Definitions of atemporal concepts are time independent. These concepts are mostly related to describing the geometry of the objects.

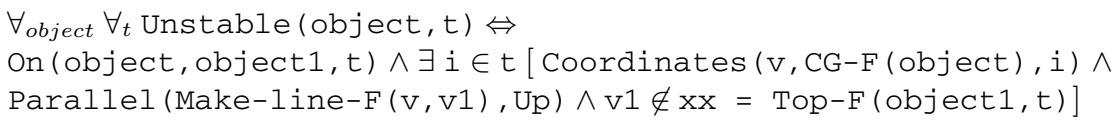

The sentence shown above in first-order logic gives the definition of an object being unstable on its place in an interval $t$. In this sentence, the relations with their names ending with $-F$ represent functions, whereas the other relations represent predicates. Each relation and constant symbol in the sentence is also defined in the framework. Below are the interpretations of these relations and symbols. 
- On (object, object $1, t)$ : is true when object is on object 1 during the interval $t$.

- Coordinates $(v$, centerofGravity, $i)$ : is true when $v$ is the vector representing the coordinates of some object's centerofGravity at an instant $i$.

- CG-F (object) : returns the vector of center of gravity of an object.

- Parallel $(1,11)$ : is true when the line 1 is parallel to the line 11.

- Make-line-F $(\mathrm{V}, \mathrm{V} 1)$ : returns a line passing through the points represented by coordinates $\mathrm{v}$ and $\mathrm{v} 1$.

- Top-F (object, $t$ ) : returns a set of points ( $x x)$ representing the top of an object in the interval $t$.

- Constant symbol Up: represents an imaginary line in the straight upward direction normal to the floor.

As a result of the interpretations given above, the predicate Unstable (object, $t$ ) is true if and only if the projection from the object's center of gravity in the straight downward direction leaves the top of the other object (on which it is places) in some instant of the interval $t$.

Here, some characteristics of the definition of Unstable should be noticed. Firstly, the definition is only describing a naive concept of an object being unstable. This definition does not represent the actual concept (as in statics or dynamics) of stability of objects. Secondly, there is a bias in the definition towards fault category location. This means that according to the definition if the object is unstable on another object, it can change its location at its own (because of gravity). Such a bias also enables easy identification of the information (regarding the naive concepts) that needs to be formalized in the form of a definition. It can also be seen that the definition of Unstable describes the concept regarding the stability of an object by considering its behavior over an extended time. And, this description is independent of any particular object. All the characteristics of the definition of Unstable mentioned above also hold for the other definitions formalized in the framework.

The framework only uses very basic concepts from geometry and mechanics (e.g. center of gravity and lines) to define the naive concepts behind the properties. This keeps the evaluation of these properties arithmetically simple and computationally effective. In the framework, the definitions of properties $d o$ represent some redundant information. For instance, according to the definitions, an unstable object is also an object that is moving. However, both these concepts are defined separately. Such redundancies are unavoidable because these are found in the naive concepts represented by the definitions. However, the redundancies have been kept from creating any inconsistency in the framework.

\section{Results}

We apply our scheme to our running example in which the die falls on the floor instead of staying on the table. In this case, after the completion of step 0 and 1(a \& b) of Fig. 2. the query generator generates two queries for the hypotheses generator. One of these queries is shown below.

showHyp ([objectID(die), place(floor), stability (stable), mobility (movable), translation(stationary), roll(rollable) ], table, gravity). 
This query consists of following three components.

1. A list of predicates, in which each predicate represents a property of the die. The arguments of the predicates show the values of respective properties of the die in its final state.

2. A value of the place (i.e. table) that was required to be achieved by the robot in its action.

3. The expected phenomena that can cause the detected fault, i.e. gravity.

A theoretical proof that as per definitions the final state of the die is represented by the values of the properties used in the query, can be found in [16]. The other query generated by the query generator only replaces the physical phenomenon of gravity with air (see step '2' in Fig. 22). It should be noticed that the query generator knows which physical phenomena should be used in the queries because it uses the ontology shown in Fig. 3. The hypotheses generator generates two separate lists of hypotheses for the phenomena of gravity (a) and air (b) in response to the posed queries.

1. State (die) :-

place (table), stability (unstable), translation (moving).

2. State (die) :-

place(floor), stability (unstable), translation(moving).

3. State (die) :-

place (floor), stability (stable), translation (stationary).

(a) Hypotheses for 'gravity'
1. State(die):-

translation (moving), place (floor).

2. State (die): -

translation (stationary), place (floor).

(b) Hypotheses for 'air'

As stated earlier, each of the generated hypotheses represents a situation that can be the cause of the detected fault. As human beings we can immediately see that hypothesis 1 for gravity, is the most relevant one for the considered scenario. Interpretation of this hypothesis gives the reason of the detected fault. According to the definitions module this interpretation reads (in natural language) as following. There existed some interval (after the release of the die) in which the die was on the table and its center of gravity was not at a fixed point in space and the projection from its center of gravity, in straight downward direction, left the top of the table in at least one of the instants of that interval. This interpretation represents the occurred situation correctly. The information obtained from the interpretation can be used by the robot to avoid the same situation in the future. In our work we assume that the interpreter interprets the hypotheses by simply selecting them one by one, however it can be seen that it is not very difficult to prune the list of the hypotheses using some heuristics before the interpretation.

We also apply our approach to a different scenario. In this scenario when the robot picks a bottle, the bottle slips from its gripper and falls on the table. Again, the detected fault is categorized under the type location. Therefore, the query generator also generates very similar queries. The only difference in the queries is that the value table is replaced by gripper.

Again, the hypothesis 1 for gravity (c) is the most relevant one for the described scenario. However, it should be noticed that if the interpretation of this hypothesis is done by using the definition of Unstable shown in section 4.3 , then this hypothesis would not represent the occurred situation correctly. Therefore, the proposed framework (discussed in section 4.3) also allows multiple definitions for a single property 
1. State (bottle) :-

place (gripper), stability (unstable), translation(moving).

2. State (bottle) :-

place (table), stability (unstable), translation (moving).

3. State (bottle) :-

place (table), stability (stable), translation (stationary).

(c) Hypotheses for 'gravity'
1. State (bottle) :translation (moving), place (table).

2. State (bottle) :translation (stationary), place (table).

(d) Hypotheses for 'air'

of objects. Although, the current framework (reported in [16]) contains only one definition for Unstable, however another definition for the same concept can easily be formed by using the insights from our work. The relations representing these different definitions can be distinguished by their names or arity. There is another important observation that can be made about the results of both the scenarios. That is, all the situations referred by the generated hypotheses can result in the respective final states of the objects in each scenario. This means that in principle all the generated hypotheses are useful for the robot. The work [16] presents the other experiments where the current approach was applied.

\section{Conclusions}

This paper is directed towards developing a strategy for service robots to successfully handle unexpected situations that occur during interactions of the robot with its dynamic environment. The idea is to utilize the existing algorithms to deal with unknown external faults in the appropriate manner. We defined four classes of these faults. In addition we proposed an approach that uses naive physics knowledge to find information regarding the situations which result in the occurrence of faults from inadequate description of planner operator class.

Application of the proposed approach to a simple pick and place scenario shows that using naive physics for unknown external fault reasoning is indeed beneficial. By using naive physics it is possible to gain useful information about the occurred fault without detailed modeling and complex computations. We find that the use of naive physics knowledge is not very complicated, because we can distribute this knowledge under different categories of faults. This distribution eases the process of formalization of the knowledge by limiting its scope. The formalization is guided by the category of the fault for which the knowledge is being formalized. By knowing the category a priori it is possible to formalize only those aspects of the knowledge which are relevant for a particular category of faults. Therefore, we propose to formalize the knowledge into different frameworks for different fault categories. We also propose that the categories of faults should be decided based on the tasks which can be performed by the robot. This means that the overall knowledge for fault reasoning also has its scope limited to a particular (type of) robot (e.g. mobile manipulators).

The future direction of the naive physics approach is to extend the ontology used in this work for other categories of faults and then formalizing more knowledge of naive physics reasoner for these categories. Another interesting issue which has been left open in this work is related to pruning the list of hypotheses generated by the hypotheses generator. Our approach can greatly benefit by developing effective methods for selecting more relevant hypotheses for interpretation. 
Lastly we may specify an enlarged set of classes with corresponding handling techniques. The new classes can originate from a design of the particular robot as well as from new realistic scenarios. As one source of scenarios we can chose the RoboCup@ Home competition where a robot has to operate in the apartment environment and perform everyday tasks. After this has been done successfully, the complete approach will contribute to the improvement of the robot's robustness towards exogenous causes of failure. These skills will make robots more adaptive and compliant with real world environments.

Acknowledgments. This work was sponsored by the B-IT foundation and the Strukturfond des Landes Nordrhein-Westfalen for female PhD students.

\section{References}

1. Nilsson, N.J.: Shakey the robot. Technical Report 323, AI Center, SRI International, 333 Ravenswood Ave, Menlo Park, CA 94025 (April 1984)

2. Pettersson, O., Karlsson, L., Saffiotti, A.: Model-free execution monitoring in behavior-based robotics. IEEE Trans. on Systems, Man and Cybernetics, Part B 37(4), 890-901 (2007)

3. Steinbauer, G.: Survey on faults of robots used in robocup (2012), http: / / www.ist.tugraz.at/rfs/

4. Gspandl, S., Pill, I., Reip, M., Steinbauer, G., Ferrein, A.: Belief management for high-level robot programs. In: 22nd International Joint Conference on Artificial Intelligence (2011)

5. Karg, M., Sachenbacher, M., Kirsch, A.: Towards expectation-based failure recognition for human robot interaction. In: 22nd International Workshop on Principles of Diagnosis (2011)

6. Sundvall, P., Jensfelt, P.: Fault detection for mobile robots using redundant positioning systems. In: IEEE International Conference on Robotics and Automation, ICRA 2006 (2006)

7. Ueda, R., Kakiuchi, Y., Nozawa, S., Okada, K., Inaba, M.: Anytime error recovery by integrating local and global feedback with monitoring task states. In: IEEE, 15th International Conference on Advanced Robotics ICAR, pp. 298-303 (2011)

8. Mendoza, J., Veloso, M., Simmons, R.: Motion interference detection in mobile robots. In: IEEE/RSJ International Conference on Intelligent Robots and Systems, IROS (2012)

9. Hayes, P.J.: The naive physics manifesto. In: Michie, D. (ed.) Expert Systems in the Micro Electronic Age, pp. 242-270. Edinburgh University Press (1979)

10. Davis, E.: The naive physics perplex. AI Magazine 19, 51-79 (1998)

11. Kleer, J.D., Brown, J.S.: A qualitative physics based on confluences, pp. 83-126. Morgan Kaufmann Publishers Inc., San Francisco (1990)

12. Davis, E.: A logical framework for commonsense predictions of solid object behaviour. Artificial Intelligence in Engineering 3(3), 125-140 (1988)

13. Kuipers, B.: Qualitative simulation. Artificial Intelligence 29, 289-338 (2001)

14. Forbus, K.D.: Qualitative process theory. Artificial Intelligence 24, 85-168 (1984)

15. Reiner, M., Slotta, J., Chi, M., Resnick, L.: Naive physics reasoning: A commitment to substance-based conceptions. Cognition and Instruction (2000)

16. Akhtar, N.: Fault reasoning based on naive physics. Technical report, Hochschule BonnRhein-Sieg, Sankt Augustin, Germany (April 2011) 